\title{
Enhancing Infrared Images Contrast for Pulsed Thermography
}

\author{
Matthieu Klein, Abdelhakim Bendada, Mariacristina Pilla*, Clemente Ibarra-Castanedo, Xavier \\ Maldague $^{++}$
}

\author{
Department of Electrical and Computer Engineering, Laval University, Quebec, (QC), Canada \\ ${ }^{++}$xavier.maldague@gel.ulaval.ca; phone 1418 656-2962; fax 1418 656-3159; mivim.gel.ulaval.ca \\ * Burns \& McDonnell, Kansas City, (MO), USA
}

\begin{abstract}
In pulsed thermography the sample to test is briefly heated with a Dirac $\delta$ heat impulse and then observed through an infrared (IR) camera recording thermal images as the sample is cooling down. The difference of temperature on the IR images between the defective areas and the sound areas is referred to as a thermal contrast [1]. The Signal-to-Noise Ratio (SNR) of such a contrast is strongly affected by the non uniformity of the initial heating impulse over the specimen. This paper describes a way to enhance this SNR by comparing the temperature evolution of each pixel of the image against the temperature evolution of a heat transfer model.
\end{abstract}

\section{Introduction}

In non destructive testing (NDT) by infrared thermography, a thermal contrast designates the difference of heat observed on the IR image between a defective area and a sound or non defective area of the specimen under inspection. A smaller contrast indicates a smaller defect or a defect that is buried deeper inside the specimen. From this definition, a simple raw IR image or thermogram can be seen as the simplest form of thermal contrast affected by a given offset. Although, when a quantitative or a better qualitative analysis is needed it is often required to further improve the thermal contrast. The absolute contrast, the running contrast, the normalized contrast and standard contrast are typical methods of computing a thermal contrast in IR thermography [1]. The drawback is that computing any of these contrasts requires knowing at least one point belonging to a sound area. It is not always possible to precisely locate such sound areas from the raw IR images in advance. This means that only reasonable assumptions can be made about the location of sound areas from the raw IR images to calculate any of the previous thermal contrast. The second issue that arises from contrast computations is the non-uniform initial heating which produces sound areas with different shifted temperature evolution. In 2001, the Differentiated Absolute Contrast or DAC solved both issues which propelled the limits of thermal contrasts in terms of quality and accuracy [2][3]. The DAC belongs to a type of extrapolated contrast (EC) that is based on the extrapolation of the temperature measured at an early time through a transient heat transfer model. This paper recalls the fundamentals of the two existing EC methods that are in turn used to introduce a third EC model that slightly improves the limits of the EC.

\section{Principle of existing extrapolated contrast (EC) methods}

In EC methods, the temperature evolutions of the sound areas are not measured. Instead, they are modeled. This is achieved by computing the temperature evolution through a thermal model. The difference between the various EC's mostly lies in the thermal models used to simulate a sound area. The sound area temperature evolution of a given point is computed starting from its initial known temperature just right after the heat impulse. In this sense, the sound area temperature evolution is an extrapolation of temperature. The temperature extrapolation starts from a real temperature measurement of the surface at the earliest time possible, at $t_{0}$ when the heat impulse occurs. In practice only the time t' slightly after $t_{0}$ is considered instead of $t_{0}$ as the heat impulse at time to saturates the camera. At this time, the temperature at the surface does not depend on subsurface defects yet, so all the surface is behaving exactly like a sound area around the time to. This allows locally predicting the expected sound area temperature at any given time after $t_{0}$ at any place, especially in defective areas. The difference between the extrapolated temperature as it is computed and the temperature as it is actually measured, gives the thermal contrast on the surface. There are currently two existing EC's.

The first $E C$ is often referred to as the $D A C$ or $E C$ with an Insulated Semi Infinite Body model (EC ISIB). It uses the assumption that a sound area can reasonably be modeled by a semi infinite body [2][3][9][10]. This assumption is quite good for most cases of specimens inspected including composite and anisotropic materials such as CFRP. The EC ISIB offers the best compromise between efficiency and complexity as it gives good practical results with a simple transient heat transfer model. 


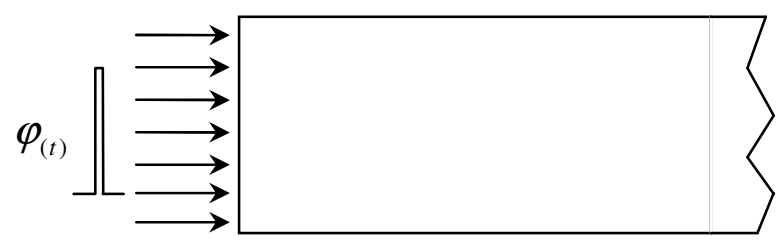

Fig. 1. Semi Infinite body thermal model as used in the DAC or EC ISIB

The sound areas model of the $E C I S I B$ uses the temperature evolution $\Delta T(t)$ at the surface $z=0$ of the specimen with the following semi infinite model:

$$
\Delta T_{I S I B}(t)=\frac{Q}{e \sqrt{\pi t}}
$$

The temperature evolution of sound areas is supposed to be close to the temperature model of a semiinfinite body, so:

$$
\Delta T_{\text {ISIB }}(t)=\frac{Q}{e \sqrt{\pi t}} \approx \Delta T_{\text {Sound_Area }_{-}}(t)
$$

Where:

$e \quad$ Thermal effusivity. $e=\sqrt{\lambda \cdot \rho \cdot c_{p}}$ in $W \cdot m^{-2} \cdot K^{-1} s^{1 / 2}$

$\alpha \quad$ Thermal diffusivity. $\alpha=\lambda /\left(\rho \cdot C_{p}\right)$ in $m^{2} \cdot s^{-1}$

$\lambda \quad$ Thermal conductivity in $W \cdot m^{-1} \cdot K^{-1}$

$\rho \quad$ Density in $\mathrm{kg} \cdot \mathrm{m}^{-3}$

$c_{p} \quad$ Specific heat capacity in $J \cdot \mathrm{kg}^{-1} \cdot K^{-1}$.

$\rho \cdot c_{p} \quad$ Volumetric heat capacity in $J \cdot m^{-3} \cdot K^{-1}$

$Q \quad$ Energy density in $J \cdot m^{-2}$

$\Delta T \quad$ Temperature change over the surface $\mathrm{z}=0$ of the material $t$ Time in s.

$h \quad$ Convective heat transfer coefficient in $W \cdot m^{-2} \cdot K^{-1}$

The temperature at the surface of the sample as measured at $t$ ', just after the input heat pulse is normally not much influenced by potential subsurface defects. The "heat wave" has not got the time at this instant to propagate deep enough into the material, thus the following practical assumption is made relatively to $t$ ':

$\Delta T_{\text {Measured }}\left(t^{\prime}\right)=\Delta T_{\text {Sound_Area }}\left(t^{\prime}\right)$

Combining Eqs. (2) and (3), it comes:

$$
\Delta T_{\text {Measured }}\left(t^{\prime}\right)=\Delta T_{I S I B}\left(t^{\prime}\right)=\frac{Q}{e \sqrt{\pi t^{\prime}}}
$$

In Eq. (4), $\Delta T_{\text {Measured }}\left(t^{\prime}\right)$ is known as it is measured, so $Q / e$ can be calculated as:

$$
\frac{Q}{e}=\Delta T_{\text {Measured }}\left(t^{\prime}\right) \cdot \sqrt{\pi t^{\prime}}
$$

Introducing $Q / e$ in Eq. (2) gives:

$$
\Delta T_{\text {Sound_Area }}(t) \approx \sqrt{\frac{t^{\prime}}{t}} \Delta T_{\text {Measured }}\left(t^{\prime}\right)
$$

As stated before, the difference between the extrapolated temperature $\Delta T_{\text {Sound_Area }}(t)$ as it is computed and the temperature $\Delta T_{\text {Measured }}(t)$ as it is measured, is the thermal contrast:

$$
\Delta T_{E C_{-} I S I B}(t)=\Delta T_{\text {Measured }}(t)-\Delta T_{\text {Sound_Area }_{-}}(t), \text { so }
$$




$$
\Delta T_{E C_{-} I S I B}(t)=\Delta T_{\text {Measured }}(t)-\sqrt{\frac{t^{\prime}}{t}} \Delta T_{\text {Measured }}\left(t^{\prime}\right)
$$

The second existing extrapolated contrast is the EC IS that still uses a 1D heat transfer model as the EC $I S I B$ described before, but it adds the thickness $L$ of the slab to inspect in consideration. The contrast is thus calculated the same way as the EC ISIB, replacing the thermal model $\Delta T_{S I B}(t)$ by $\Delta T_{I S}(t)$ where IS stands for Insulated Slab [8]. The boundary conditions remain adiabatic as it is the case for the EC ISIB. This implies the medium eventually tends to a steady state temperature greater than its environment. Obviously the $E C$ IS works better for contrast computations for flat plate samples that are insulated or have rather low thermal losses. The $\Delta T_{I S}(t)$ transient heat transfer equation can be solved through various methods. The thermal quadrupoles method in which the time space is represented in a Laplace domain will be used as an example [4][8]. The inverse Laplace transform is numerically computed at the very end.

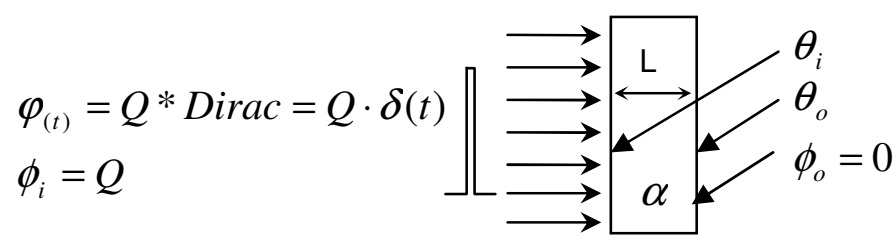

Fig. 2. EC using a thermal model of an insulated slab in Laplace space

In reference to the model in figure 2, let $L\left(\Delta T_{I S}(t)\right)=\theta_{i}(p)$ be the Laplace transform of the temperature function at the surface and let $L(\varphi(t))=\phi_{i}(p)=Q$ be the Laplace transform of the heat flux density function. $p$ is the Laplace variable. In this case, the thermal quadrupole equation is [4]:

$$
\left(\begin{array}{l}
\theta_{i} \\
\phi_{i}
\end{array}\right)=M *\left(\begin{array}{l}
\theta_{o} \\
\phi_{o}
\end{array}\right) \text { with } M=\left(\begin{array}{ll}
A & B \\
C & D
\end{array}\right)
$$

where $\theta_{i}$ represents the input Laplace temperature on the front face of the slab and $\theta_{o}$ is the output Laplace temperature on the rear face. From the quadrupole theory, the $M$ quadrupole matrix for a solid slab is:

$$
\begin{array}{ll}
A=D=\cosh (k L) & C=\lambda k \cdot \sinh (k L) \\
B=\sinh (k L) /(\lambda k) & k=\sqrt{p / \alpha}
\end{array}
$$

Knowing that $\phi_{i}=L\left(\varphi_{(t)}\right)=Q$ and that $\phi_{o}=0$ because the rear face of the plate is adiabatic, it comes:

$$
\theta_{i}=Q A / C=\frac{Q}{e} \cdot \frac{\operatorname{coth} \sqrt{p L^{2} / \alpha}}{\sqrt{p}}=\frac{Q}{e} \cdot \theta_{i}{ }^{\prime}
$$

The same principle as the EC ISIB is applied to Eq. (8). This way Eq. (2) can be written as follows:

$$
\Delta T_{I S}(t)=\left.L^{-1}\left(\theta_{i}\right)\right|_{t}=\left.\frac{Q}{e} L^{-1}\left(\theta_{i}^{\prime}\right)\right|_{t} \approx \Delta T_{\text {Sound_Area }_{\text {Are }}}(t)
$$

Since Eq. (3) is true for all EC methods, Eq. (4) can be rewritten as:

$$
\Delta T_{\text {Measured }}\left(t^{\prime}\right)=\Delta T_{I S}\left(t^{\prime}\right)=\left.\frac{Q}{e} L^{-1}\left(\theta_{i}^{\prime}\right)\right|_{t^{\prime}}
$$

In Eq. (10), $\Delta T_{\text {Measured }}\left(t^{\prime}\right)$ is known as it is measured so $Q / e$ can be calculated again. Q/e is then introduced in (9):

$$
\Delta T_{\text {Sound_Area }}(t) \approx \frac{\left.L^{-1}\left(\theta_{i}^{\prime}\right)\right|_{t}}{\left.L^{-1}\left(\theta_{i}^{\prime}\right)\right|_{t^{\prime}}} \Delta T_{\text {Measured }}\left(t^{\prime}\right)
$$

The $E C$ IS is the difference between the measured temperature and the temperature computed without defect, hence:

$$
\Delta T_{E C_{-} I S}(t)=\Delta T_{\text {Measured }}(t)-\frac{\left.L^{-1}\left(\theta_{i}^{\prime}\right)\right|_{t}}{\left.L^{-1}\left(\theta_{i}{ }^{\prime}\right)\right|_{t^{\prime}}} \Delta T_{\text {Measured }}\left(t^{\prime}\right)
$$


Unlike the $E C I S I B$, the $E C$ IS needs at least the plate thickness $L$ and the thermal diffusivity as input parameters. If not known, it is possible to found them interactively through a manual curve fitting for example with IR View [6]. Although such a curve fitting does require the knowledge of a sound area which hinders the benefits of EC contrast methods, it is a valuable way to determine thermal parameters from calibrated samples.

\section{Extrapolated Contrast using thickness and thermal losses}

The EC IS described above needs the thickness and is considered as perfectly adiabatic. The last $E C$ as proposed in this paper is not adiabatic anymore. A Non Insulated Slab model $\Delta T_{N I S}(t)$ now replaces $\Delta T_{I S}(t)$ and takes the thermal losses from the convective effect into account as depicted in Figure 3.

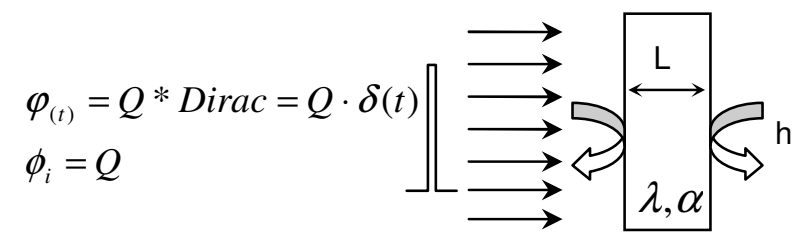

Fig. 3. EC using a thermal model acting as a non insulated slab (NIS)

To find $L\left(\Delta T_{N I S}(t)\right)=\theta_{i}(p)$, the matrixes $\mathrm{BC} 1$ and $\mathrm{BC} 2$ are added to the previous thermal quadrupole system [4]:

$$
\left(\begin{array}{c}
\theta_{i} \\
\phi_{i}
\end{array}\right)=B C 1 * M * B C 2 *\left(\begin{array}{l}
\theta_{o} \\
\phi_{o}
\end{array}\right)
$$

The thermal losses are represented by the Boundary Conditions (BC1 and $\mathrm{BC} 2)$ matrixes respectively that characterize each side of the flat plate under inspection. Thermal losses by convection are supposed to be the same on both sides, so [4]:

$$
B C 1=B C 2=\left[\begin{array}{cc}
1 & 1 / h \\
0 & 1
\end{array}\right]
$$

where $h$ is the convective heat transfer coefficient. This can be simplified as:

$$
\left(\begin{array}{l}
\theta_{i} \\
\phi_{i}
\end{array}\right)=M^{\prime} *\left(\begin{array}{l}
\theta_{o} \\
\phi_{o}
\end{array}\right) \text { with } M^{\prime}=\left(\begin{array}{ll}
A^{\prime} & B^{\prime} \\
C^{\prime} & D^{\prime}
\end{array}\right)
$$

Knowing that $\phi_{i}=L\left(\varphi_{(t)}\right)=Q$ and that $\phi_{o}=0$ because there is no excitation on the rear face, it comes:

$$
\begin{aligned}
& \theta_{i}=Q A^{\prime} / C^{\prime}=Q \cdot \theta_{i}{ }^{\prime \prime} \text { where } \\
& \theta_{i}{ }^{\prime \prime}=\frac{h s / \lambda k+c}{2 c h+\lambda k s+h^{2} s / \lambda k} \text { with } c=\cosh (k L) \text { and } s=\sinh (k L)
\end{aligned}
$$

Note that $\theta_{i}{ }^{\prime \prime}$ is different from $\theta_{i}{ }^{\prime}$ calculated in Eq. (8). In particular, $\theta_{i}{ }^{\prime \prime}$ contains the thermal conductivity $\lambda$. The same principle as used on the EC ISIB from Eq. (2) is applied to Eq. (16), so:

$$
\Delta T_{N I S}(t)=\left.L^{-1}\left(\theta_{i}\right)\right|_{t}=\left.Q \cdot L^{-1}\left(\theta_{i}^{\prime \prime}\right)\right|_{t} \approx \Delta T_{\text {Sound_Area }}(t)
$$

Eq. (3) remains true through the definition of the $E C$ methods, so that Eq. (4) is now:

$$
\Delta T_{\text {Measured }}\left(t^{\prime}\right)=\Delta T_{N I S}\left(t^{\prime}\right)=\left.Q \cdot L^{-1}\left(\theta_{i}{ }^{\prime \prime}\right)\right|_{t^{\prime}}
$$

In Eq. (18) $\Delta T_{\text {Measured }}\left(t^{\prime}\right)$ is known as it is measured so $Q$ can be calculated and introduced in (17):

$$
\Delta T_{\text {Sound_Area }}(t) \approx \frac{\left.L^{-1}\left(\theta_{i}^{\prime \prime}\right)\right|_{t}}{\left.L^{-1}\left(\theta_{i}^{\prime \prime}\right)\right|_{t^{\prime}}} \Delta T_{\text {Measured }}\left(t^{\prime}\right)
$$
defect:

The $E C$ is the difference between the measured temperature and the temperature computed without

$$
\Delta T_{E C_{-} N I S}(t)=\Delta T_{\text {Measured }}(t)-\frac{\left.L^{-1}\left(\theta_{i}{ }^{\prime \prime}\right)\right|_{t}}{\left.L^{-1}\left(\theta_{i}{ }^{\prime \prime}\right)\right|_{t^{\prime}}} \Delta T_{\text {Measured }}\left(t^{\prime}\right)
$$


The EC NIS requires the knowledge and/or adjustment of the thermal conductivity and losses in addition of all the parameters of the ECIS.

\section{Normalization}

All the EC's images have flat sound areas even when the initial heat impulse is not uniform. Areas other than sound areas, i.e. the defective areas or non-zero contrast areas show various amplitudes of contrast. It must be enlightened that any non-zero contrast area still carries a hidden non uniformity due to a potential non uniform heating impulse. A way to correct this non uniformity is to divide $\Delta T_{E C_{-} X}(t)$ by $\Delta T_{\text {Measured }}\left(t^{\prime}\right)$ where $X$ is ISIB, IS or NIS. In this case, Eq. (20) becomes:

$$
\Delta T_{\text {Normilized_EC_NIS }}(t)=\frac{\Delta T_{\text {Measured }}(t)}{\Delta T_{\text {Measured }}\left(t^{\prime}\right)}-\frac{\left.L^{-1}\left(\theta_{i}{ }^{\prime \prime}\right)\right|_{t}}{\left.L^{-1}\left(\theta_{i}{ }^{\prime \prime}\right)\right|_{t^{\prime}}}
$$

This does not make the image looking visually better but makes it righter from both a quantitative and qualitative point of view.

\section{Implementation}

Both $\Delta T_{E C_{-} I S}(t)$ and $\Delta T_{E C_{-} N I S}(t)$ require a numerical inverse Laplace transform in order to calculate the temperature. Stehfest's algorithm is a simple but yet powerful way to numerically inverse the Laplace transform [4].

The full implementation of all of the three EC methods was carried out in IR View, a Matlab program with a graphical user interface for basic Infrared image processing. IR View is used within the Multipolar Infrared Vision Group (MIVIM) as general purpose tool in research and inspection [5], [6]. It also allows to quickly accomplish standard image processing such as pulsed phase thermography [1], as well as fundamental image enhancement tools such as smoothing IR images, adjusting the color map, viewing the temperature evolution in time, computing contrasts, extracting an image or a whole processed sequence or simply navigating through the sequence of IR images. IR View is free and open source under the GNU License and can be downloaded from the web site of MIVIM [5].

The Extrap CT (Extrapolated Contrast) button is selected meaning IR View is computing and displaying a contrast image in its display window.

This $3^{\text {rd }}$ IR View window allows adjusting the various parameters of the three Extrapolated Contrast such as the Initial Time that is referred to as $t$ ' in the previous explanations. It also enables changing the diffusivity, plate thickness, conductivity and thermal losses. The effect of moving the cursors in the contrast image is shown in real time. From a practical point of view, it is difficult to fine tune the EC parameters without a graphical interface.

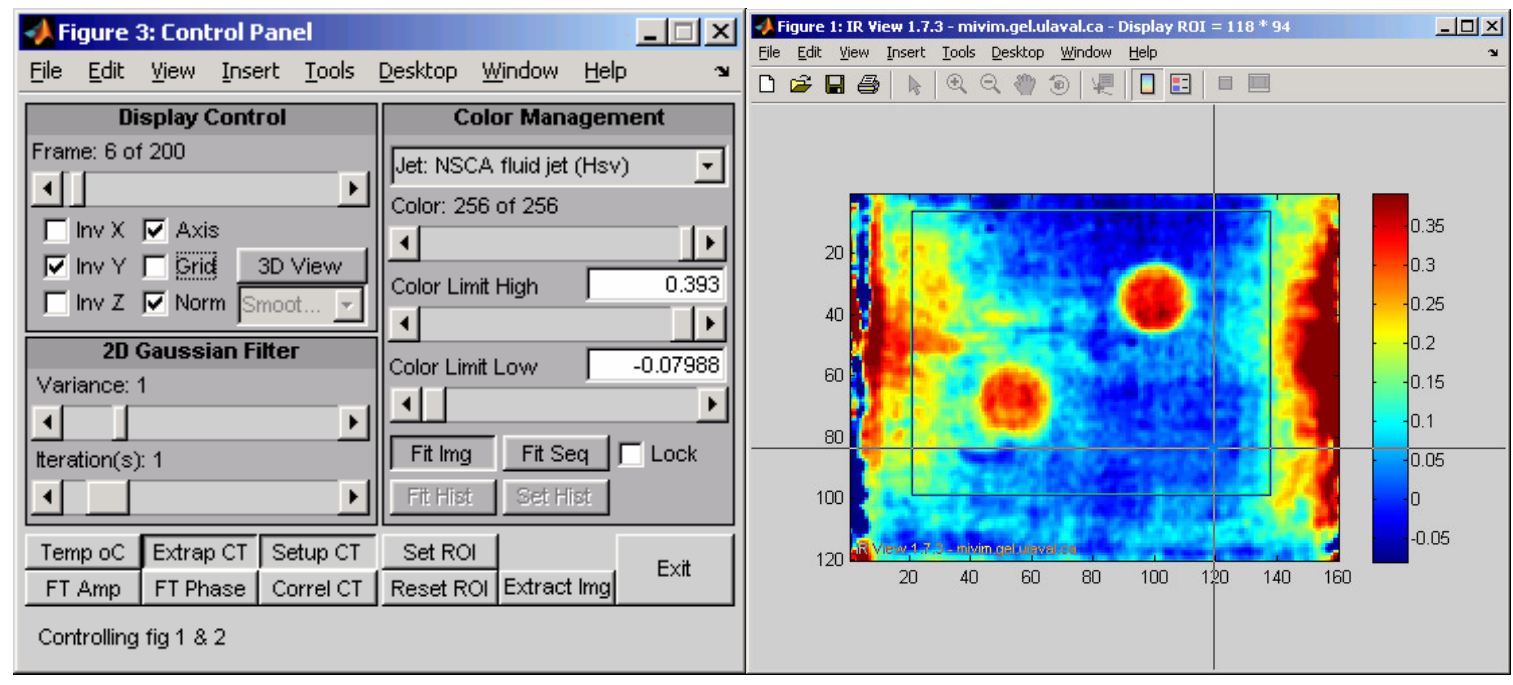

Fig. 4. The main window of (left) and the Display window (right) of IR View 


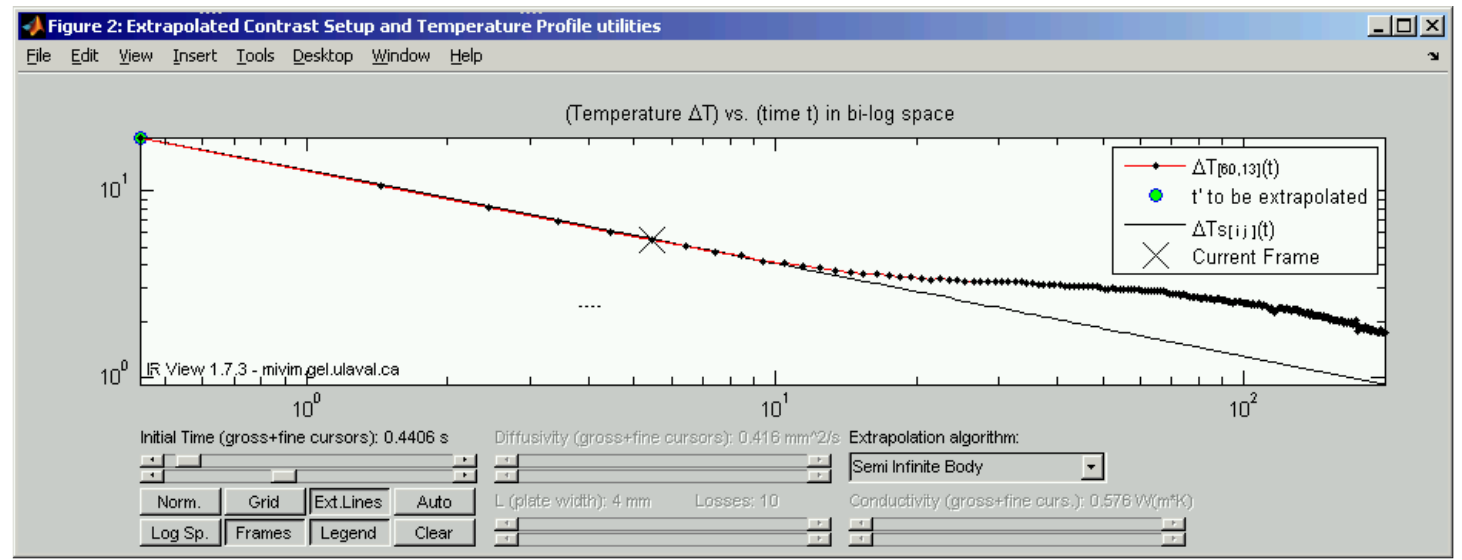

Fig. 5. The EC control window of IR View

\section{Experimental results}

The experimental results help to understand the rational, advantage and limits of the EC NIS. The sample tested is a CFRP plate with three artificial defects buried inside as illustrated in Figure 6.

The infrared sequence has the following specification:

Thermal Excitation:

Number of Frames:

2 ms heat pulses from 2 photographic flashes

Time of first frame after the Dirac heat pulse:

Time of the last frame:

Time between each frame:

Resolution:

200

$0.05 \mathrm{~s}$

$19.95 \mathrm{~s}$

$0.1 \mathrm{~s}$.

$160 \times 120 \times 12 b p p$

Figure 7 shows the best image from a visual point of view that could be obtained: from a smoothed raw image showing the temperature increase in ${ }^{\circ} \mathrm{C}$ (left), from a PPT phase sequence (center) and from a correlation image (right) [7]. Figure 8 shows the three contrasts namely the DAC/EC ISIB (Figure 8a), the EC IS (Figure $8 b$ ), and the $E C N I S$ (Figure 8c),. Each figure contains 3 views. The top left view shows 2 curves. One of the curves is $\Delta T_{\text {Measured }}(t)$ of a known and arbitrary sound area. The second curve is $\Delta T_{\text {Sound Area }}(t)$, the extrapolation of the temperature that is computed accordingly to the 3 models presented in this paper. In clear the top left view shows $\Delta T_{\text {Measured }}(t)$ and $\Delta T_{\text {Sound_Area }}(t)$. The smaller difference between those 2 curves, the better, as it means the model fits the free defect temperature evolution better. This is a requirement for the EC contrast to work correctly as shown in Eq. (3). Recall the sound area temperature is computed when calculating an $E C$ because it is not measurable in areas where there are defects, nor it can be approximated from other areas because the initial thermal excitation is usually not uniform, which would result in unwanted thermal offsets in the contrast image. The black cross on the top left view of the 3 Figure 8 shows which image of the sequence is currently processed. The cross corresponds to image \#5 in all three cases. The top right view shows the resulting contrast image at frame 5 out of 200 , i.e. at $0.65 \mathrm{sec}$ after the Dirac heat impulse. The box inside the IR image is a Region of Interest (ROI). The color palette is linearly mapped between the minimum and maximum value contained within this ROI. This allows a better color mapping. The bottom view shows the contrast of a point (pixel) over the time over both a defective and sound area. The contrast of the sound area is always the lower curve. The curve with a spike is typical from a defective area and the spike corresponds to the maximum contrast. 


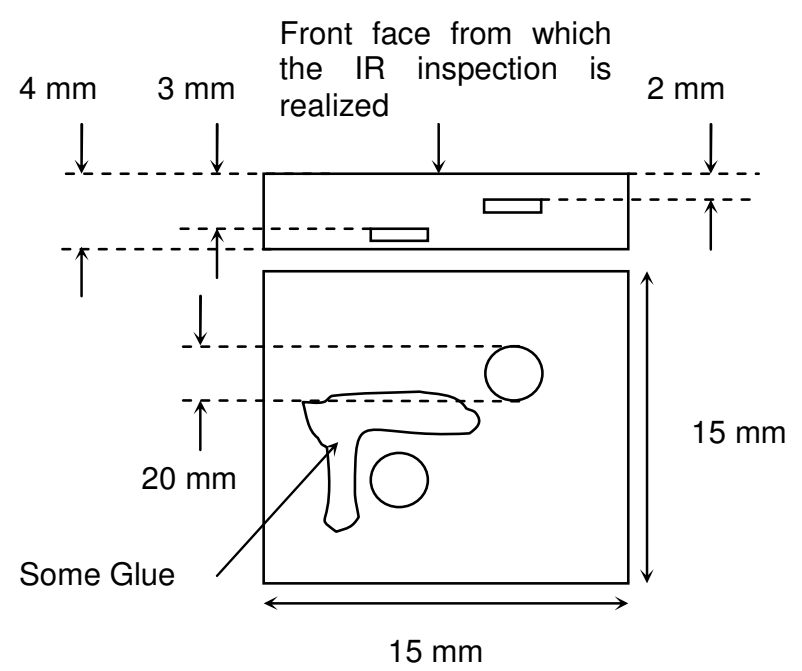

Fig. 6. CRFP plate slab sample with 3 known defects (1 glued area +2 circular fluorocarbon resin implants)
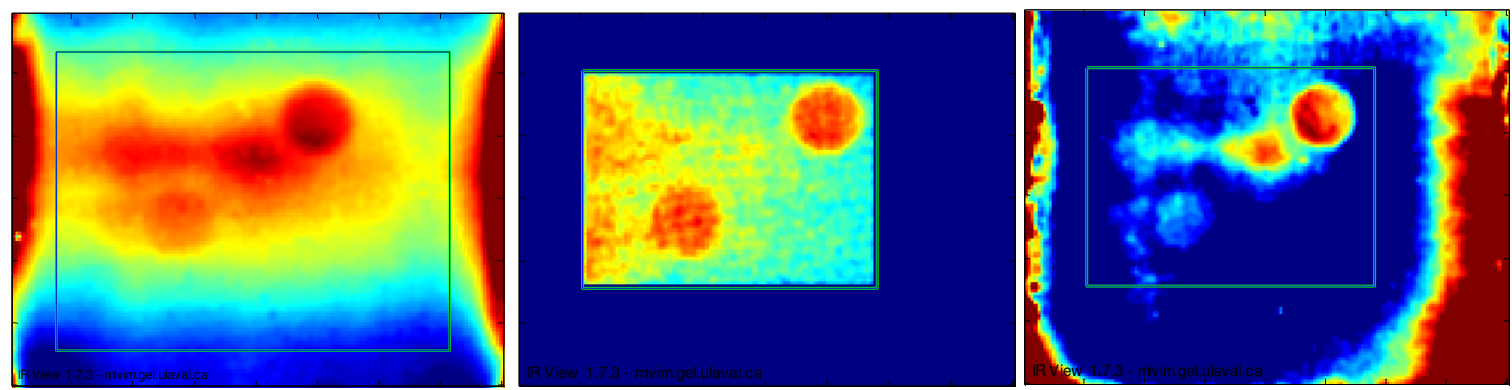

Fig. 7. Standard processing techniques. The box corresponds to a Region of Interest (ROI) in which the full color map is applied
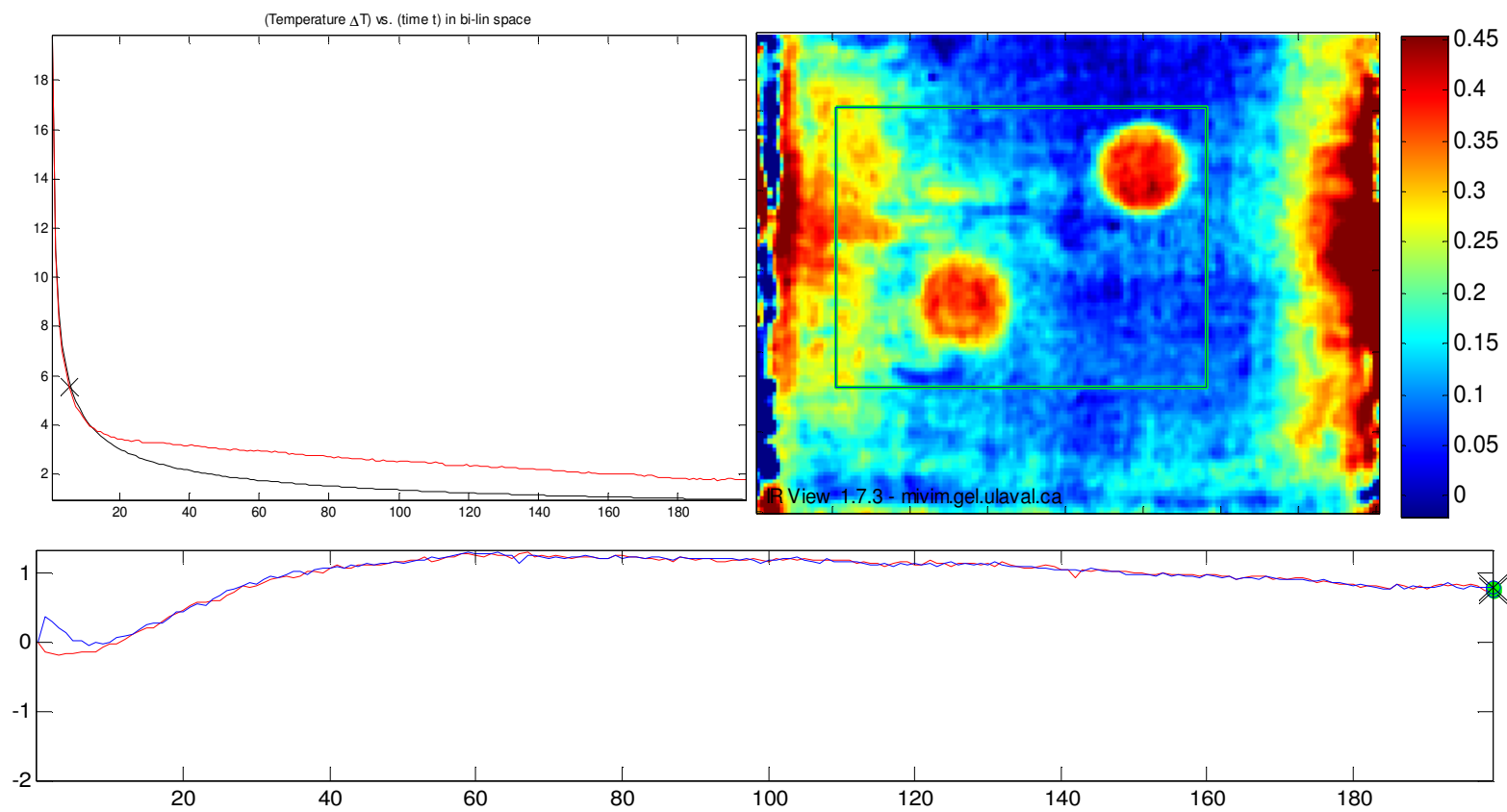

Fig. 8.a. $E C I S I B$ 

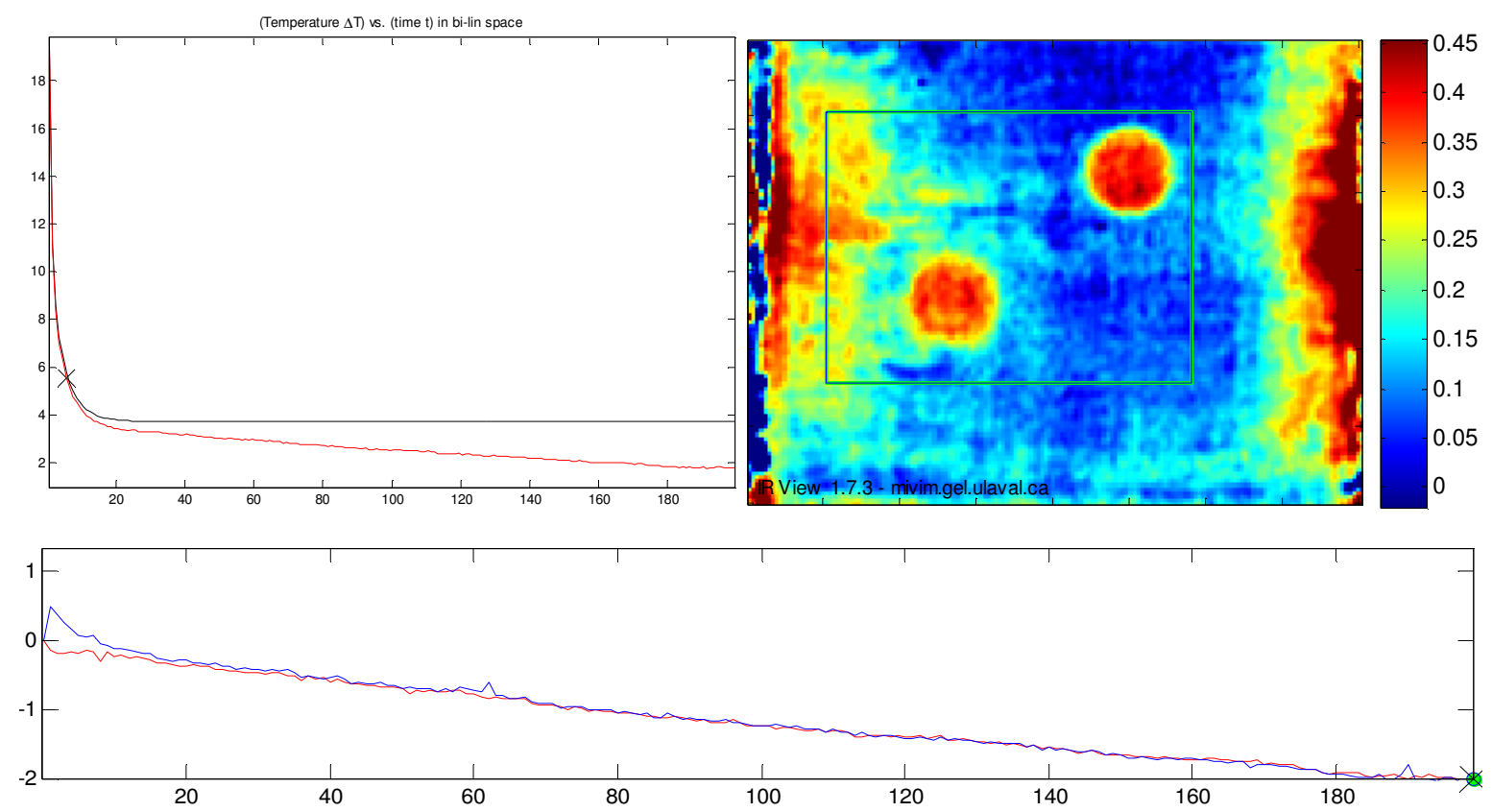

Fig. 8.b. EC IS
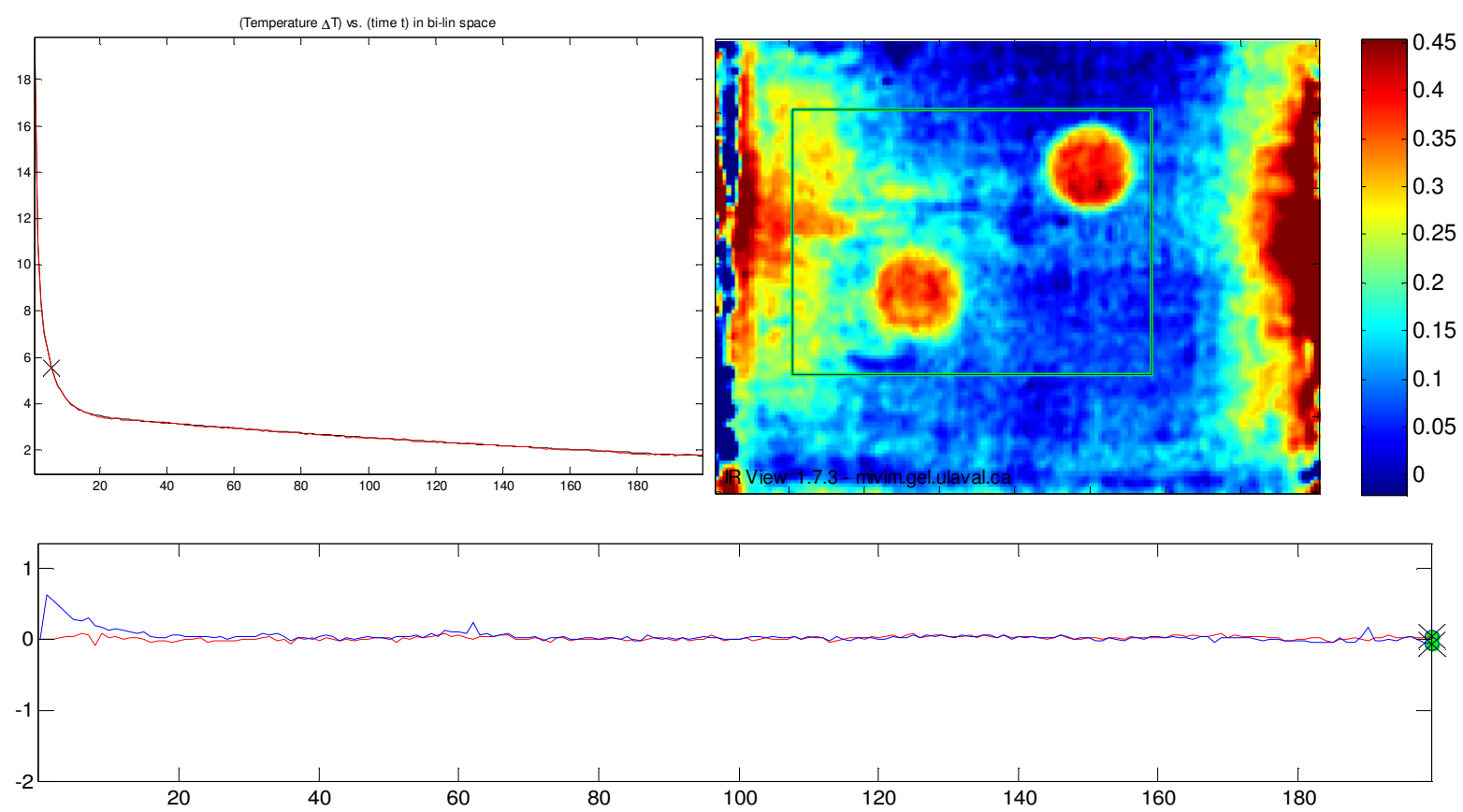

Fig. 8.c. EC NIS

The first observation is that the non uniformity effect is reduced in the three EC images when compared with the raw thermogram shown in Fig 7 (left). The second observation is that the EC images are visually not much different from each other at this instant. This is explained by the fact the maximum contrast of defects always occurs at early times, i.e. before the effects of the back side. There would be a greater difference if the defects were closer to the back side though. Thus, even the EC ISIB that uses the assumption of a semi infinite body still shows a quite good contrast image. In the three bottom views of Fig 8.a, 8.b and 8.c, the contrast looks identical between the fame 1 and 10. After frame 10, both the EC ISIB and EC IS give a bigger error whereas the contrast should be and remain close to 0 . Only the EC NIS shows a steady contrast close to 0 after the frame $\# 10$. 

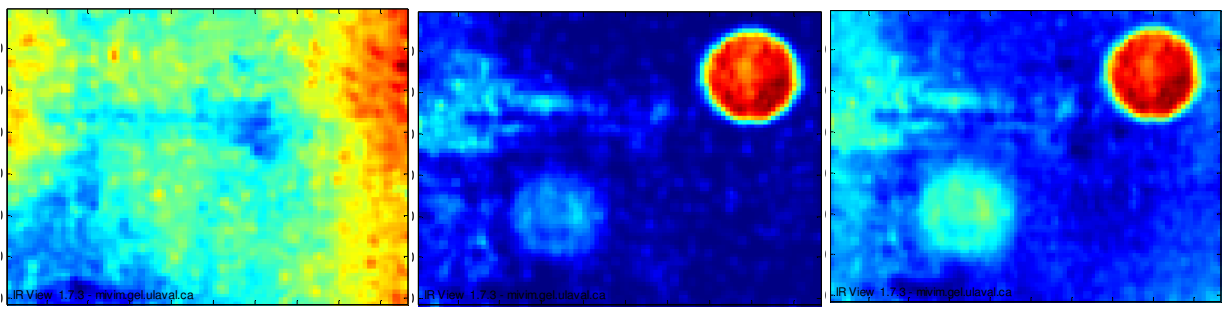

Fig. 11. Maximum contrast images for the EC ISIB, EC IS, EC NIS respectively taken on the whole sequence (frames \#1 to \#200)

This explains why the maximum contrast image of Figure 11 (left) do not clearly show any of the defects with the EC ISIB when computed between frame \#1 and frame \#200. Since the ECISIB, and to some extend the $E C I S$, provide unsuitable results at later times, the maximum contrast image gets affected in turn if a long sequence is taken in account for the maximum contrast computation. The EC NIS presents the most suitable result. It is possible to obtain a maximum contrast image that is almost that good with the EC ISIB if the maximum contrast image is computed within a shorter time frame but this would requires knowing where to start and end the sequence.

\section{Conclusion}

This study validates the relevance of the EC ISIB for the case of Teflon inserts in CFRP. Although the EC $I S I B$ is not accurate at later times, subsurface defects will always have their maximum contrast occurring before the EC ISIB loses its accuracy. The EC ISIB is convenient because it doesn't require the knowledge of any thermal specification such as thermal losses, diffusivity or conductivity. Improving the heat transfer model with thickness or both thickness and thermal losses do improve the contrast at later times. This is of interest for small defects that are very close the back side of the slab or in case one wishes to compute the maximum contrast images on the whole sequence of images, but at the cost of adjusting or knowing extra thermal parameters. Nothing is free.

\section{Acknowledgements}

The financial support of NSERC and of the Research Chairs of Canada is acknowledged.

\section{REFERENCES}

[1] X. Maldague, "Theory and Practice of Infrared Technology for Non Destructive Testing", Wiley, New York, 2001.

[2] C. Ibarra-Castanedo, D. González, M. Klein, M. Pilla, S. Vallerand and X.Maldague, "Infrared image processing and data analysis," Infrared Physics \& Technology Vol. 46, Issues 1-2, December 2004, pp.75-83

[3] M. Pilla, M. Klein, X. Maldague A. Salerno, "New absolute contrast for pulsed thermography" in D. Balageas G. Busse, C. Carlomagno (Eds.), Proc. QIRT (Quantitative Infrared Thermography), Dubrovnik, Croatia, 2002

[4] D. Maillet et al., Thermal Quadrupole, Wiley \& Sons, England, 2000, ISBN, 0-471-98320-9

[5] Multipolar Infrared Vision, http://mivim.gel.ulaval.ca/

[6] Matthieu Klein, Clemente Ibarra-Castanedo, Xavier Maldague, Hakim Bendada, "IR-View: a simple infrared images viewer for Infrared Thermography”, SPIE, Thermosense XXX, V. Vavilov, D. D. Burleigh eds., SPIE vol. 6939, March 2008

[7] Klein, Clemente Ibarra-Castanedo, Hakim Bendada, Xavier Maldague, "IR image sequence processing through correlation operator for pulsed thermography," SPIE, Thermosense XXX, V. Vavilov, D. D. Burleigh eds., SPIE vol. 6939, March 2008

[8] Hernan Dario Benitez, Clemente Ibarra-Castanedo, Abdel Hakim Bendada, Xavier Maldague, Humberto Loaiza and Eduardo Caicedo, "Modified Differential Absolute Contrast Using Thermal Quadrupoles for the Nondestructive Testing of Finite Thickness Specimens by Infrared Thermography", in CCECE 2006 Canadian Conference on Electrical and Computer Engineering, (Ottawa (Ontario) Canada), May 7-10 2006.

[9] BOSCHER D.M., BALAGEAS D.L., DEOM A.A. and GARDETTE G., "Non destructive evaluation of carbon expoxy laminates using transient infrared thermography ", Proceedings 16 th NDE Symposium, San Antonio (TX), USA, April 21-23, 1987.

[10] DEOM A., BOSCHER D., and BALAGEAS D.L., "Pulsed photothermal non destructive testing. Application to carbon expoxy laminates", Review of Progress in Quantitative Non Destructive Evaluation, vol. 9A, p. 525-531, 1990. 
http://dx.doi.org/10.21611/qirt.2008.08_02_03 